\title{
Barrier height formation in organic blends/metal interfaces: Case of tetrathiafulvalene-tetracyanoquinodimethane/Au(111)
}

\author{
José I. Martínez,, ${ }^{1,2, a)}$ Enrique Abad, ${ }^{3}$ Juan I. Beltrán, ${ }^{2}$ Fernando Flores, ${ }^{2}$ \\ and José Ortega ${ }^{2}$ \\ ${ }^{1}$ Depto. Superficies y Recubrimientos, Instituto de Ciencia de Materiales de Madrid (CSIC), \\ ES-28049 Madrid, Spain \\ ${ }^{2}$ Depto. Física Teórica de la Materia Condensada and Condensed Matter Physics Center (IFIMAC), \\ Universidad Autónoma de Madrid, ES-28049 Madrid, Spain \\ ${ }^{3}$ Institute of Theoretical Chemistry, Universität Stuttgart, D-70569 Stuttgart, Germany
}

(Received 10 July 2013; accepted 15 November 2013; published online 4 December 2013)

\begin{abstract}
The interface between the tetrathiafulvalene/tetracyanoquinodimethane (TTF-TCNQ) organic blend and the $\mathrm{Au}(111)$ metal surface is analyzed by Density Functional Theory calculations, including the effect of the charging energies on the molecule transport gaps. Given the strong donor and acceptor characters of the TTF and TCNQ molecules, respectively, there is a strong intermolecular interaction, with a relatively high charge transfer between the two organic materials, and between the organic layer and the metal surface. We find that the TCNQ LUMO peak is very close to the Fermi level; due to the interaction with the metal surface, the organic molecular levels are broadened, creating an important induced density of interface states (IDIS). We show that the interface energy level alignment is controlled by the charge transfer between TTF, TCNQ, and Au, and by the molecular dipoles created in the molecules because of their deformations when adsorbed on $\mathrm{Au}(111)$. A generalization of the Unified-IDIS model, to explain how the interface energy levels alignment is achieved for the case of this blended donor/acceptor organic layer, is presented by introducing matrix equations associated with the Charge Neutrality Levels of both organic materials and with their intermixed screening properties. ( 2013 AIP Publishing LLC. [http://dx.doi.org/10.1063/1.4836635]
\end{abstract}

\section{INTRODUCTION}

In the last years, the field of organic electronics has received a lot of attention from research and industry because of its potential use in electronic devices. ${ }^{1-3}$ For the Surface Science Community, the study of the conductivity of these systems is very challenging, ${ }^{2,4}$ these properties depending crucially on the different interface barriers formed at organic/organic or at metal/organic contacts. ${ }^{3,5,6}$ For the electronic industry, these materials provide a cheap and environment-friendly way to obtain electronic devices as an alternative to conventional semiconductors. However, these materials have the drawback that their electron mobility is very low, due to the molecular character of the crystals and its weak intermolecular interaction.

The analysis of different metal/homogeneous organic interfaces has been studied by a wide number of theoretical and experimental groups (e.g., see Refs. 7-17). As a result of this work, it seems fair to conclude that the organic/metal energy level alignment at the contact is the result of different mechanisms operating at the interface: charge transfer between the materials; compression of the metal electron tails due to Pauli repulsion (so called "pillow" effect); orientation of molecular dipoles; and, for reactive interfaces, formation of gap states in the organic materials. For non-reactive interfaces, the first three mechanisms (charge transfer, "pillow" effect and molecular dipoles) can be described together

\footnotetext{
a) joseignacio.martinez@icmm.csic.es
}

by means of a Unified-Induced Density of Interface States (Unified-IDIS) mode $1^{17}$ based on the concept of the Charge Neutrality level $(C N L)$, an interface screening parameter $(S)$ and the "pillow" and molecular dipoles. . $^{13,15-17}$

One advantage of using organic semiconductors is the possibility of combining different organic materials to control and adjust the electronic properties of the barrier heights at metal/organic interfaces. In particular, donor-acceptor/metal interfaces are very important in organic devices such as solar cells. ${ }^{18}$ Different organic donor-acceptor/metal interfaces have recently been explored; we mention here the work on (TTF-TCNQ)/Au(111) interfaces, ${ }^{19-21}$ as well as the work by El-Sayed et al. on CuPc-PFP/Au(111) and $\mathrm{Cu}(111)$, and $\mathrm{F}_{16} \mathrm{CuPc}-\mathrm{PEN} / \mathrm{Au}(111)$ and $\mathrm{Cu}(111) .{ }^{18}$

From a fundamental point of view, as well as for the applications, it is very important to understand the effect of the donor-acceptor and molecule-metal interactions on the position of the organic energy levels with respect to the metal Fermi level $\left(E_{F}\right)$. The theoretical analysis of the energy level alignment in these interfaces is a challenging task due to problems such as the large sizes of these systems, the deficiencies of standard DFT techniques to properly describe the organic energy levels at metal/organic interfaces, ${ }^{3}$ as well as the intrinsic complexity of these multi-component organic/metal interfaces.

In this paper we address theoretically this problem by studying the interesting heterogeneous (TTF-TCNQ)/ $\mathrm{Au}(111)$ interface. This interface combines two organic molecules with strongly acceptor (TCNQ) and donor (TTF) 
characters, this fact highlighting the possible effects associated with the difference between the electro-negativities of the organic molecules. The TTF-TCNQ crystal has been widely studied because it was the first organic conductor; ${ }^{4}$ it has also been suggested as a good candidate for organic electronic devices due to its high mobility. The interface between the TTF and TCNQ organic crystals has been analyzed recently; ${ }^{22,23}$ these studies show that the interface is metallic ${ }^{22}$ due to the overlap (and charge transfer) between the density of states corresponding to the TTF highest occupied molecular orbital (HOMO) and the TCNQ lowest unoccupied molecular orbital (LUMO) levels. ${ }^{23}$

The first step in the analysis of the electronic structure and energy level alignment for the (TTF-TCNQ)/Au(111) interface is to obtain a reliable interface geometry; this is discussed in Sec. II A. Once the geometry has been determined, the interface electronic structure is analyzed in Sec. II B including in our DFT calculation the effects associated with the molecule charging energy that will allow us to correct appropriately the transport energy gap of both organic materials. ${ }^{24-29}$ In Sec. III we present a generalization of the Unified-IDIS model for the interface between a blended organic layer and a metal, introducing matrix equations for the organic blends that allow us to extend to this case the concepts used previously for a homogeneous organic layer. In particular, the screening parameter $S$ is represented now by a $2 \times 2 S$ matrix. Finally, in Sec. IV we present our conclusions.

\section{THE (TTF-TCNQ)/Au(111) INTERFACE}

We analyze the (TTF-TCNQ)/Au(111) interface in two steps. First, we determine the adsorption geometry by means of DFT calculations that include van der Waals interactions. Once the geometry of the interface has been obtained, we analyze the electronic properties of this complex interface using a local-orbital DFT approach that we have found more convenient for this purpose ${ }^{24-29}$ in these calculations appropriate corrections are introduced to take care of the main deficiencies of the DFT calculation.

\section{A. (TTF-TCNQ)/Au (111): Interface geometry}

In this work we have analyzed the interface geometry suggested by González-Lakunza et al. $;^{19}$ in this geometry the TTF and TCNQ molecules lie parallel to the Au(111) surface, forming alternating rows of TTF and TCNQ molecules. In our calculations we have initially placed the molecules on the $\mathrm{Au}(111)$ surface following this pattern, and then the atomic geometry has been fully relaxed by means of DFT calculations. In spite of the strong donor-acceptor character of the TTF-TCNQ molecules, van der Waals interactions may be important in order to determine their adsorption geometry. ${ }^{30}$ Thus, we have used the DFT + D approach $^{31}$ as implemented in the Quantum Espresso code. ${ }^{32}$ For this purpose, we have used the revised version of the generalized gradient corrected approximation of Perdew, Burke, and Ernzerhof (rPBE), ${ }^{33}$ and an empirical efficient van der Waals (vdW) $\mathrm{R}^{-6}$ correction to add dispersive forces

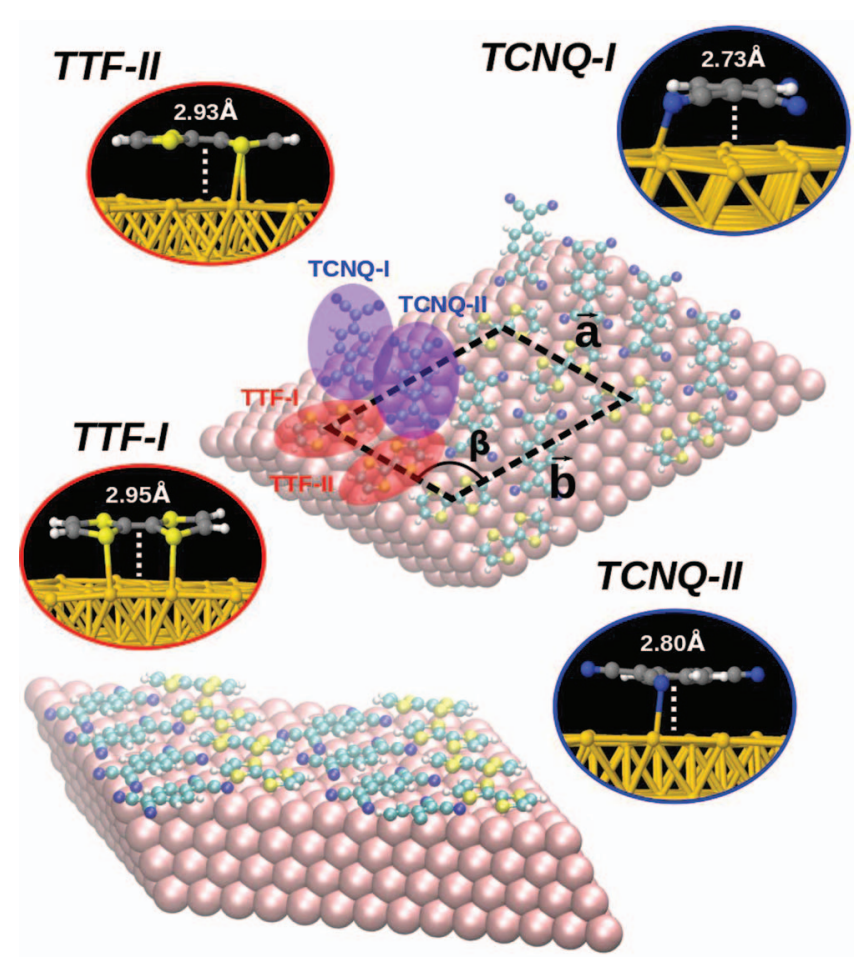

FIG. 1. Adsorption geometry for the (TTF-TCNQ) organic layer on $\mathrm{Au}(111)$ : top and side views. Black dashed lines in the top view indicate the unit cell used in all the calculations. The adsorption geometry for each of the 4 organic molecules in the unit cell is shown in more detail in the oval insets.

to conventional density functionals (DFT - D) ${ }^{34}$ In this method, the vdW correction is added to the DFT total energy by the expression $E_{v d W}=\sum_{i, j} \frac{C_{i j}}{R_{i j}^{6}} f\left(R_{i j}\right)$, where $C_{i j}$ and $R_{i j}$ are the vdW coefficients and the distance between atom $\mathrm{i}$ and $\mathrm{j}$, respectively. The vdW coefficients can be calculated as described by Elstner $e t a l .{ }^{35} f\left(R_{i j}\right)$ is a damping function which prevents a divergence in the energy as $R_{i j}$ tends to zero as $f\left(R_{i j}\right)=\left(1-\exp \left[-3.0\left(\frac{R_{i j}}{R_{0 i j}}\right)^{7}\right]\right)^{4}$, where $R_{0 i j}$ is the sum of atomic van der Waals radii. They can be calculated from the vdW radii provided by Gavezzotti and co-workers. ${ }^{36}$

Lattice vectors and unit cell for the periodic DFT calculation are shown (dashed black line) in top panel of Fig. 1 $\left(\mathbf{b}=20.49 \AA ; \mathbf{a}=14.64 \AA ; \beta=120^{\circ}\right)$. The $\mathrm{Au}(111)$ surface is represented by a slab with $4 \mathrm{Au}$ layers and the (TTF-TCNQ) adlayer is placed on one side of the slab; thus, there are 2 TTF and 2 TCNQ molecules per unit cell. In total, the unit cell contains 208 atoms. The Brillouin zone (BZ) has been sampled by means of a $[2 \times 4 \times 1]$ Monkhorst-Pack grid, ${ }^{37}$ guaranteeing a full convergence in energy and density.

The relaxed interface geometry is shown in Figure 1. The adsorption geometry for the two TTF molecules in the unit cell is slightly different. The TTF-I molecule is tilted, i.e., the $\mathrm{S}$ atoms on one side of the molecule are closer to the surface than the $\mathrm{S}$ atoms on the other side (see inset in Figure 1); the $\mathrm{S}$-Au distance of the closer $\mathrm{S}$ atoms is $d(\mathrm{~S}-\mathrm{Au})=2.65 \AA$. For TTF-II a different pair of $\mathrm{S}$ atoms is closer to the Au surface (see corresponding inset in Figure 1) with a distance of $d(\mathrm{~S}-\mathrm{Au})=2.57 \AA$. For both TTF molecules the distance 
from the central $\mathrm{C}$ atoms to the Au surface is very similar, $\mathrm{d}_{\perp} \sim 2.94 \AA$. In the case of the TCNQ molecules the main feature is that some of the $\mathrm{N}$ atoms are bonded to the Au surface. The TCNQ-I molecule (see inset) presents a bent geometry with $3 \mathrm{~N}$ atoms forming bonds with $\mathrm{Au}$ atoms on the surface, with $\mathrm{N}-\mathrm{Au}$ distances of $d(\mathrm{~N}-\mathrm{Au}) \sim 2.38 \AA$. In the case of the TCNQ-II molecule (see inset) one $\mathrm{N}$ atom forms a strong bond to $\mathrm{Au}$, with $d(\mathrm{~N}-\mathrm{Au})=2.19 \AA$. The distances of the central $\mathrm{C}$ atoms to the Au surface are $\mathrm{d}_{\perp}=2.73 \AA$ (TCNQ-I) and $\mathrm{d}_{\perp}=2.80 \AA$ (TCNQ-II).

\section{B. (TTF-TCNQ)/Au (111): Interface electronic structure}

In a second step we analyze the electronic structure and energy level alignment for the (TTF-TCNQ)/Au(111) interface geometry presented in Sec. II A. Due to the complexity of this analysis we have used a computationally efficient approach, ${ }^{23-29}$ based on local-orbital DFT in which appropriate corrections are introduced to take care of the main inaccuracies of the DFT calculation.

For this purpose we use the efficient local-orbital DFT code FIREBALL. ${ }^{38-41}$ This technique is based on a local-orbital formulation of DFT in which self-consistency is implemented on the orbital occupation numbers; ${ }^{42}$ the orbital occupation numbers have been obtained using the orthonormal Löwdin orbitals. ${ }^{38,41}$ In these calculations we have used a basis set of optimized $s p^{3} d^{5}$ numerical atomic orbitals (NAOs) ${ }^{43}$ for Au, C, N, and S, and $s$ for $\mathrm{H}$, with cut-off radii (in a.u.): $s=4.5, p=4.9$, and $d=4.3(\mathrm{Au}) ; s=4.0$, $p=4.5$, and $d=5.4(\mathrm{C}) ; s=3.6, p=4.1$, and $d=5.2(\mathrm{~N}) ; s$ $=4.2, p=4.7$, and $d=5.5(\mathrm{~S})$; and $s=4.1(\mathrm{H}) .{ }^{45}$ This is the same basis set as used in our previous works involving the TCNQ/Au(111) ${ }^{28}$ and TTF/Au(111) ${ }^{29}$ interfaces. In order to determine accurately the interface energy level alignment, the main error introduced by the non-completeness of the localorbital basis set, once the geometry is fixed (see Sec. II A), appears on the relative initial position of the molecular and metal levels, ${ }^{29}$ and this is corrected in our approach as discussed below, e.g., see Eq. (1b). In these calculations we have used the Local Density Approximation (LDA) functional ${ }^{40}$ and the ion-electron interaction is modeled by means of norm-conserving scalar-relativistic pseudopotentials. ${ }^{44}$

For organic materials, it is well-known that in standard DFT calculations the Kohn-Sham energy levels do not properly describe the electronic energy levels of the system and transport gaps are usually too small., 36,47 For example, the experimental gap between the ionization and the affinity levels of the gas-phase TCNQ (TTF) molecule is around 5.3 (6.3) eV, while the energy gap between the Kohn-Sham HOMO and LUMO levels in LDA or generalized gradient approximation (GGA) calculations are $1.6(2.6) \mathrm{eV}^{23}$ This problem is related to the molecule self-interaction energy as described by the molecule charging energy $U .^{3,46,47}$

The effect of the molecule-metal interaction on the electronic structure of the molecules is threefold: ${ }^{46}$ (1) the coupling of the molecular orbitals with delocalized states in the metal broadens the molecular levels into resonances; (2) the charge rearrangement due to the molecule-metal interaction creates a potential between molecule and metal that shifts the molecular levels relative to the metal Fermi energy; and (3) the dynamical polarization response of the metal to added electrons or holes in the molecule (i.e., the image potential). This is a correlation effect that opposes the self-interaction correction and tends to reduce the energy gap (i.e., the metal polarization response reduces the value of the charging energy of the molecule $U) ., 46,47$

The effects of the self-interaction energy and metal dynamical polarization response on the interface electronic structure are included in our calculations in a practical and simplified way introducing for each molecule a scissor operator:

$$
O_{\alpha}^{\text {scissor }}=\frac{U_{\alpha}}{2} \sum_{(\mu \nu)}\left\{\left|\mu_{i}\right\rangle\left\langle\mu_{i}|-| v_{i}\right\rangle\left\langle v_{i}\right|\right\},
$$

where $\left|\mu_{i}\right\rangle$ and $\left|v_{i}\right\rangle$ being the empty (occupied) orbitals of the isolated molecule (with the actual geometry of the molecule on the surface). The charging energy for each molecule, $U_{\alpha}$, is taken from previous calculations, ${ }^{28,29}$ since the average metalmolecule distances in those works are similar to the ones obtained in our actual geometry. We also introduce for each molecule a rigid shift of the molecular levels by means of a shift operator:

$$
O_{\alpha}^{\text {shift }}=\varepsilon_{\alpha} \sum_{(\beta)}|\beta\rangle\langle\beta|,
$$

where $|\beta\rangle$ being the orbitals for each isolated molecule. Using these operators, we can fix in our local-orbital DFT calculations the initial value of the HOMO-LUMO gap and midgap position relative to the metal Fermi energy, for the TTF and TCNQ molecules on $\mathrm{Au}(111)$. Also, with these operators we correct the main inaccuracies introduced in the DFT calculation for the (TTF-TCNQ)/Au(111) electronic structure (e.g., due to the LDA functional and local-orbital basis set). In particular, the initial HOMO, LUMO, and midgap position relative to the metal Fermi level are shown in Figure 2(right). The values of the mid-gap positions for the TTF and TCNQ molecules are obtained from accurate $\Delta$-SCF calculations ${ }^{23}$ for single molecules with the geometry of the adsorbed molecules as obtained in Sec. II A. In
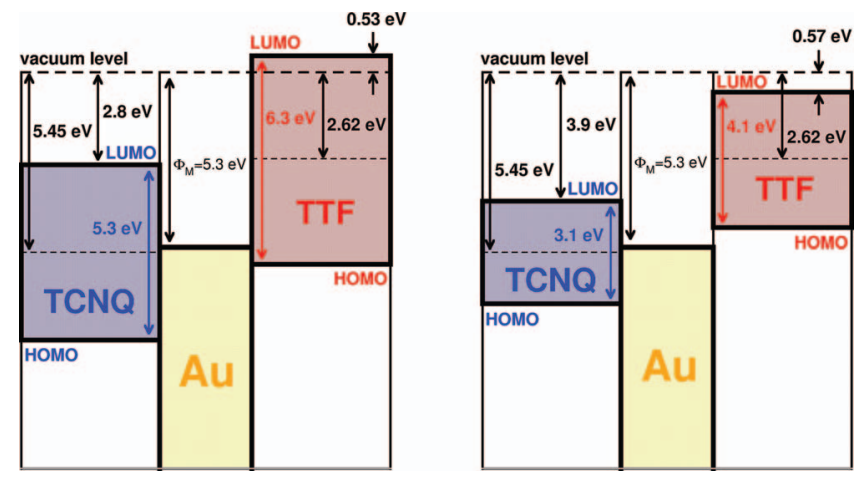

FIG. 2. Energy levels positions for the gas-phase (left panel) TTF and TCNQ molecules with respect to the $\mathrm{Au}(111)$ surface. The right panel shows how dynamical polarization or screening effects at the interface reduce the transport gaps for TTF and TCNQ, shifting in different directions occupied and empty states (see text). ${ }^{3}$ 
practice we have taken an average of the two molecules on the unit-cell since the mid-gap positions obtained for both molecules is similar. For the values of the HOMO-LUMO gaps for TTF and TCNQ at the (TTF-TCNQ)/Au(111) interface we have taken the values obtained in previous calculations for the TCNQ/Au(111) $)^{28}$ and TTF/Au(111) ${ }^{29}$ interfaces. This is a reasonable approximation since the adsorption distance is similar in both cases. Notice that in this approximation the dynamical polarization response of the system is entirely due to the metal surface ${ }^{28,29}$ and we have neglected possible changes in the HOMO-LUMO gap due to changes in the geometry of the molecules.

The broadening of the molecular states due to their coupling with metal states and the effect of the electrostatic potentials due to charge transfer at the interface are then calculated in an approximate way using the localorbital DFT calculations mentioned above, corrected using Eqs. (1a) and (1b). ${ }^{23-29}$

Figure 3 shows the electron density of states (DOS) for the (TTF-TCNQ)/Au(111) interface projected onto the orbitals of the two molecules, as well as the sum of both density of states profiles. In the same figure, we also show the energy levels of the isolated (but deformed) molecules; for comparison, the transport gaps of these isolated molecules have been corrected using the same values of $U_{\alpha}$ introduced for the calculation of the (TTF-TCNQ)/Au(111) interface. In this figure the Fermi energy, $E_{F}$, as well as the LUMO and HOMO levels of both molecules, are shown. Notice that the main effect of the contact is to broaden the molecular levels, creating an important induced density of interface states (clearly seen in the TTF or the TCNQ energy gaps) and a shift of the molecular levels with respect to $E_{F}$ due to the induced potential on each molecule.

The results shown in Fig. 3 can be compared with the experimental evidence for this interface. ${ }^{19,20}$ The experimen-

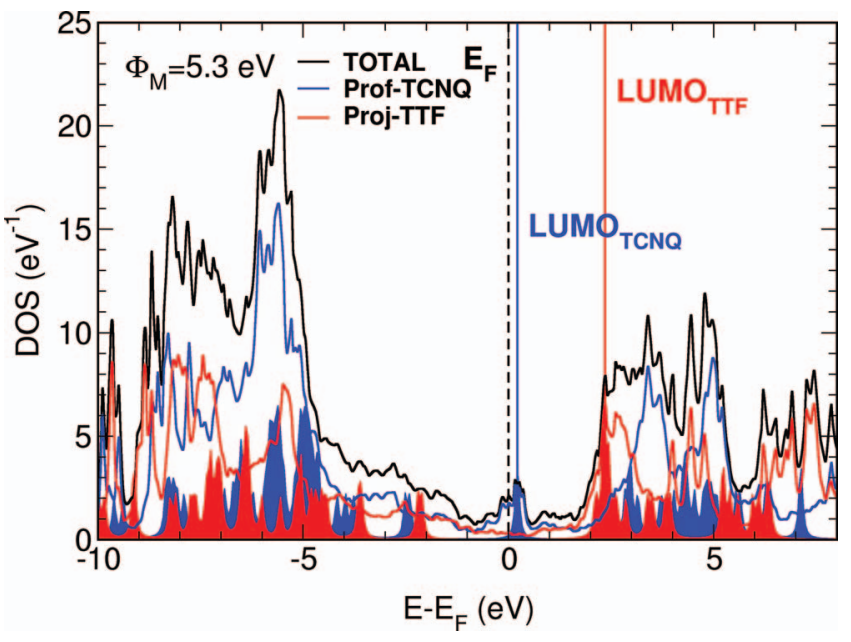

FIG. 3. Projected density of states (in $\mathrm{eV}^{-1}$ ) onto the TTF and TCNQ molecules for $\Phi_{\mathrm{M}}=5.3 \mathrm{eV}$ in the (TTF-TCNQ)/Au(111) interface referred to the Fermi energy. (Black solid line) Total DOS profile; (blue and red solid lines) TCNQ and TTF contributions, respectively; and (blue and red shaded regions) energy levels (with a lorentzian broadening) for the TCNQ and TTF gas-phase molecules (see text), respectively, shifted by the corresponding $V^{t o t}$. tal spectroscopy data show that the TTF LUMO peak is $1.7 \mathrm{eV}$ above $E_{F},{ }^{19}$ to be compared with our theoretical value of $2.3 \mathrm{eV}$ above $E_{F}$. On the other hand, a Kondo resonance associated with the TCNQ LUMO level has been observed in this system by Fernández-Torrente $e t a l .{ }^{20}$ suggesting that the TCNQ LUMO level, as calculated in our DFT approach, should be located close to the Fermi level, in good agreement with our calculations. We should also mention that other structures found in the spectroscopy of González-Lakunza et $a l .{ }^{19}$ have been shown by these authors to be associated with metal surface states resonances. Thus, we conclude that the results shown in Fig. 3 are in good agreement with the experimental evidence. ${ }^{19,20}$

In order to obtain an appropriate description of the interaction between the metal and the organic molecules, we have found convenient to analyze the same interface changing fictitiously the metal work-function $\Phi_{\mathrm{M}}\left(=-E_{F}\right)$. This can be achieved in our calculation by using the shift operator of Eq. (2). Top panel of Fig. 4 shows our results for the excess of charge, $\delta n$, found for the metal and the molecules, and bottom panel of Fig. 4 the potentials induced in TTF and TCNQ molecules w.r.t. the metal as a function of the fictitious metal
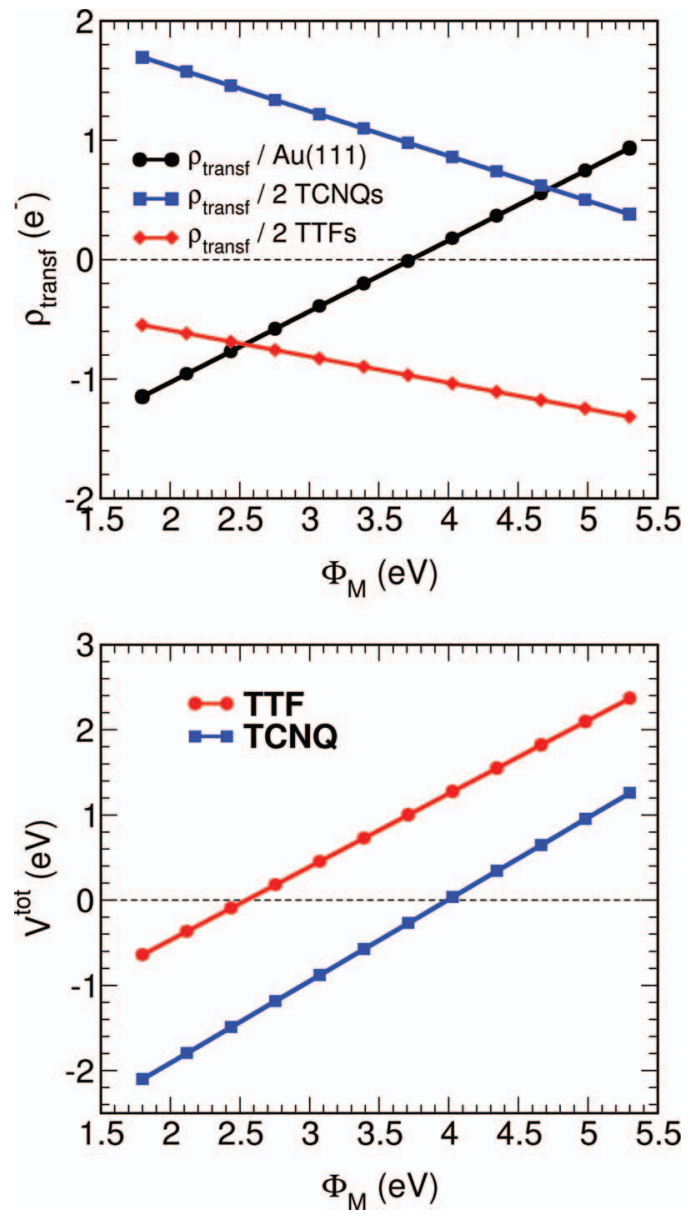

FIG. 4. DFT results for the charge transfer and IDIS potential as a function of a fictitious change in the metal work function, see text. (Top panel) Net charge transfer between TTF (red), TCNQ (blue), and $\mathrm{Au}(111)$ (black) per unit cell in the (TTF-TCNQ)/Au(111) interface; the unit cell contains 2 TTF and 2 TCNQ molecules. (Bottom panel) IDIS potential for the TTF and TCNQ molecules. 
work-function. For $\Phi_{\mathrm{M}}=5.3 \mathrm{eV}$ (Au work-function) we find that $\delta n_{T C N Q}=0.19$ electrons $/$ molecule, $\delta n_{T T F}=-0.61$ electrons/molecule, and $\delta n_{A u}=0.42$ electrons/(pair of TTF and TCNQ molecules). At the same time, we find that the TCNQ (TTF) levels are shifted by $1.26(2.37) \mathrm{eV}$ with respect to the metal (this positive sign means that the molecular levels are shifted downwards in energy), in such a way that the LUMO level of TCNQ is very close to $E_{F}$ (initially, this LUMO is $1.4 \mathrm{eV}$ above $E_{F}$, see Fig. 2).

\section{IDIS MODEL ANALYSIS}

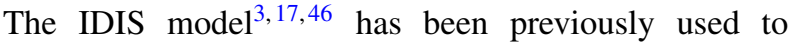
analyze different Metal-Organic ${ }^{24-29}$ or Organic-Organic ${ }^{23}$ interfaces; in all these cases the organic layers are homogeneous, i.e., contain only one kind of organic molecule. In this section we extend the IDIS-model for the case of an organic layer with two different types of molecules and apply it to the analysis of the (TTF-TCNQ)/Au(111) interface (in this discussion, we neglect the small differences between the same kind of molecules in the unit cell). The discussion presented here also shows how this analysis can be generalized for a more complex organic layer.

\section{A. Discussion of the general model}

In a first step, we relate the induced charges on the organic molecules, $\delta n_{T T F}$ and $\delta n_{T C N Q}$, to the total induced potentials on each molecule, $V^{t o t} T_{T F}$ and $V^{t o t}{ }_{T C N Q}$, by means of the following equations: ${ }^{26,29,46,48}$

$$
\begin{gathered}
\delta n_{T T F}=\left(C N L_{T T F}-E_{F}-e V_{T T F}^{t}\right) D_{T T F}, \\
\delta n_{T C N Q}=\left(C N L_{T C N Q}-E_{F}-e V_{T C N Q}^{t}\right) D_{T C N Q},
\end{gathered}
$$

here $C N L_{T T F}$ and $C N L_{T C N Q}$ are the charge neutrality levels of both organic materials, and $D_{T T F}$ and $D_{T C N Q}$ their corresponding average density of states around the Fermi level $\left(E_{F}=-\Phi_{M}\right)$; notice that the initial value of $\left(C N L_{i}-E_{F}\right)$ is reduced by the induced potential, $e V_{i}^{\text {tot }}$, which shifts the organic levels w.r.t. the metal Fermi energy (for $e V_{i}^{t o t}$ positive, the corresponding organic levels are shifted in the downwards direction).

We can also relate the potentials, $V^{t o t} T_{T F}$ and $V^{t o t}{ }_{T C N Q}$, to the charges, $\delta n_{T T F}$ and $\delta n_{T C N Q}$, by the equations:

$$
\begin{gathered}
e V_{T T F}^{\text {tot }}=U_{T T F}^{e f f} \delta n_{T T F}+J^{e f f} \delta n_{T C N Q}+e V_{T T F}^{\text {tot }(0)}, \\
e V_{T C N Q}^{\text {tot }}=U_{T C N Q}^{e f f} \delta n_{T C N Q}+J^{e f f} \delta n_{T T F}+e V_{T C N Q}^{\text {tot }(0)},
\end{gathered}
$$

where $U_{i}^{e f f}$ is a kind of charging energy for the corresponding molecule (including all the equivalent molecules of the monolayer) and $J^{e f f}$ is an effective interaction, mainly electrostatic, between TTF and TCNQ. ${ }^{26,46} e V_{i}^{\text {tot( }(0)}$ represents the potential induced in the $i$-molecule when $\delta n_{T T F}=\delta n_{T C N Q}=0$; this is created, in our case, by the molecular intrinsic dipoles of the two molecules (which is mainly due to $\mathrm{TCNQ}^{28}$ ).

It is also convenient to combine Eqs. (2a), (2b), (3a), and (3b) by introducing $\delta n_{T T F}-$ Eq. (2a) - and $\delta n_{T C N Q}-$ Eq. (2b)
- into the Eqs. (3a) and (3b). This yields the following matrix equations:

$\left[\begin{array}{c}e V_{T T F}^{t o t} \\ e V_{T C N Q}^{t o t}\end{array}\right]=(\mathbf{1}-\mathbf{S})\left[\begin{array}{c}C N L_{T T F}-E_{F} \\ C N L_{T C N Q}-E_{F}\end{array}\right]+\mathbf{S}\left[\begin{array}{c}e V_{T T F}^{t o t(0)} \\ e V_{T C N Q}^{t o t(0)}\end{array}\right]$,

where

$\mathbf{S}=(\mathbf{1}+\mathbf{U D})^{-1}=\left[\begin{array}{cc}1+U_{T T F}^{\text {eff }} D_{T T F} & J^{\text {eff }} D_{T C N Q} \\ J^{\text {eff }} D_{T T F} & 1+U_{T C N Q}^{\text {eff }} D_{T C N Q}\end{array}\right]^{-1}$

We can also rewrite the matrix Eq. (4a) as follows:

$$
e \boldsymbol{V}^{\text {tot }}=(\mathbf{1}-\mathbf{S})\left(\mathbf{C N L}-\boldsymbol{E}_{\boldsymbol{F}}-e \boldsymbol{V}^{\text {tot }(0)}\right)+e \boldsymbol{V}^{\text {tot }(0)} \text {. }
$$

Equations (4) and (5) generalize to the heterogeneous monolayer the equations for an homogeneous one, ${ }^{26,46}$ in this simple case, we have the following scalar equations:

$$
\begin{aligned}
e V^{t o t} & =(1-S)\left(C N L-E_{F}\right)+S e V^{t o t(0)} \\
& =(1-S)\left(C N L-E_{F}-e V^{t o t(0)}\right)+e V^{t o t(0)},
\end{aligned}
$$

where the term, $(1-S)\left(C N L-E_{F}-e V^{t o t(0)}\right)$ yields the induced potential associated with the charge transfer between the metal and the organic semiconductor, $S=1 /$ $\left(1+U^{\text {eff }} D\right)$ being the interface screening parameter. Notice that Eqs. (4) and (5) can be interpreted in the same way using $2 \times 2$-matrices that incorporate the effect of both organic materials. It is interesting to realize that for $J^{e f f}=0$, Eq. (5) decouples into the equations for two independent monolayers; this indicates that $J^{\text {eff }}$ provides the coupling between the two organic materials. On the other hand, if we take $C N L_{T T F}=C N L_{T C N Q} ; D_{T T F}=D_{T C N Q} ; U_{T T F}^{e f f}=U_{T C N Q}^{e f f}$; and $e V_{T C N Q}^{\text {tot }(0)}=e V_{T T F}^{\text {tot }(0)}$ we also recover the monolayer case with an effective $U$ given by $U^{e f f}+J^{e f f}$.

\section{B. The blended interface (TTF-TCNQ)/Au(111)}

From Eqs. (2a) and (2b) and the results shown in Figure 4, we can calculate the values of $D_{T T F}, D_{T C N Q}, C N L_{T T F}$, and $C N L_{T C N Q}$; this fitting yields: $D_{T T F}=0.78 \mathrm{eV}^{-1}, D_{T C N Q}$ $=4.64 \mathrm{eV}^{-1}, C N L_{T T F}=-2.09 \mathrm{eV}$, and $C N L_{T C N Q}=-4.08 \mathrm{eV}$ (the energy origin is the vacuum level), see Table I. These values of $D_{i}$ are in good agreement with the ones shown in Fig. 3; notice also that for TCNQ (TTF) the $C N L$ is $0.18 \mathrm{eV}$ $(1.52 \mathrm{eV})$ below the LUMO level.

Equations (4a) and (4b) can also be used to calculate $U_{T T F}^{e f f}, U_{T C N Q}^{e f f}$, and $J^{\text {eff }}$, as well as $e V_{T C N Q}^{\text {tot }(0)}$ and $e V_{T T F}^{\text {tot }(0)}$. This fitting yields $U_{T T F}^{e f f}=4.5 \mathrm{eV} ; U_{T C N Q}^{e f f}=4.0 \mathrm{eV}$;

TABLE I. Values of D (in $\left.\mathrm{eV}^{-1}\right),\left(C N L-E_{F}\right), U^{\text {eff }}, J^{\text {eff }}$, and $e V^{\text {tot }}(0)$ (all in eV) for TTF and TCNQ in the (TTF-TCNQ)/Au(111) interface, see text. $\left(C N L-E_{F}\right)$ is the initial position of the CNL w.r.t. the Fermi level of the metal, see Fig. 2(right). D is an average induced density of states around $\mathrm{E}_{\mathrm{F}}$, see Fig. 3.

\begin{tabular}{lccccc}
\hline \hline & $D\left(\mathrm{eV}^{-1}\right)$ & $C N L-E_{F}$ & $U^{\text {eff }}$ & $J^{\text {eff }}$ & $e V^{\text {tot }(0)}$ \\
\hline TTF & 0.78 & 3.21 & 4.5 & 1.93 & -0.22 \\
TCNQ & 4.64 & 1.22 & 4.0 & 1.93 & +0.75 \\
\hline \hline
\end{tabular}


$J^{\text {eff }}=1.93 \mathrm{eV} ; e V_{T T F}^{\text {tot }(0)}=-0.22 \mathrm{eV}$; and $e V_{T C N Q}^{\text {tot }(0)}=0.75$ $\mathrm{eV}$. We should comment that for calculating $U_{i}^{\text {eff }}$ and $J^{\text {eff }}$, we have also analyzed the case of the TTF (TCNQ) layer on $\mathrm{Au}(111)$, eliminating the TCNQ (TTF) rows while keeping for the remaining TTF (TCNQ) rows the geometry of the full heterogeneous monolayer. From these calculations, we obtain for the TTF (TCNQ) layer the following values for the charge transfer (2 molecules): $\rho_{\text {transf }}=-1.02 \mathrm{e}^{-}\left(-0.02 \mathrm{e}^{-}\right)$for $\Phi_{\mathrm{M}}$ $=5.3 \mathrm{eV}$, and $\rho_{\text {transf }}=-0.12 \mathrm{e}^{-}\left(1.78 \mathrm{e}^{-}\right)$for $\Phi_{\mathrm{M}}=1.8 \mathrm{eV}$; and the induced potential: $V^{\text {tot }}=2.2 \mathrm{eV}(0.91 \mathrm{eV})$ for $\Phi_{\mathrm{M}}$ $=5.3 \mathrm{eV}$, and $V^{t o t}=0.15 \mathrm{eV}(-2.69 \mathrm{eV})$ for $\Phi_{\mathrm{M}}=1.8 \mathrm{eV}$, which allow us to calculate $U_{T T F}$ and $U_{T C N Q}$. All these results are summarized in Table I.

In our case we obtain the following quantities: $\left(1+U_{T T F}^{\text {eff }} D_{T T F}\right)=4.51, \quad J^{\text {eff }} D_{T C N Q}=8.95, \quad J^{\text {eff }} D_{T T F}$ $=1.50$, and $\left(1+U_{T C N Q}^{e f f} D_{T C N Q}\right)=19.56$. Then,

$$
\mathbf{1}-\mathbf{S}=\left[\begin{array}{ll}
0.74 & 0.12 \\
0.02 & 0.94
\end{array}\right]
$$

Equations (5) and (7) embody the main effects found in our DFT-based calculations. Notice first that from these equations we obtain the following relations: $\Delta V_{T T F}^{t o t}=0.86 \Phi_{M}$ and $\Delta V_{T C N Q}^{\text {tot }}=0.96 \Phi_{M}$, in good agreement with Fig. $4(\Delta$ defines the difference between the corresponding values for $\Phi_{\mathrm{M}}$ equal to 5.3 and $1.8 \mathrm{eV}$, remember $\Phi_{\mathrm{M}}=-\mathrm{E}_{\mathrm{F}}$, in other words, these equations define the slopes in bottom panel of Fig. 4). On the other hand, we find, by neglecting the coupling between the two organic molecules, that

$$
\begin{gathered}
e V_{T T F}^{t o t} \cong 0.74\left(C N L_{T T F}-E_{F}-e V_{T T F}^{t o t(0)}\right)+e V_{T T F}^{t o t(0)}, \\
e V_{T C N Q}^{t o t} \cong 0.94\left(C N L_{T C N Q}-E_{F}-e V_{T C N Q}^{t o t(0)}\right)+e V_{T C N Q}^{\text {tot }(0)},
\end{gathered}
$$

which can be interpreted, in a first approximation, as the independent behavior of each organic submonolayer (a similar result is obtained taking $J^{\text {eff }}=0$ in Eqs. (3) and (4)). The TTF-TCNQ interaction introduces the changes $0.12\left(C N L_{T C N Q}-E_{F}-e V_{T C N Q}^{t o t(0)}\right)$ and $0.02\left(C N L_{T T F}-E_{F}-e V_{T T F}^{t o t(0)}\right)$ in $e V^{t o t} T T F$ and $e V^{t o t} T C N Q$, respectively. The effect of the $\left(C N L_{T C N Q}-E_{F}-e V_{T C N Q}^{\text {tot }(0)}\right)$ on $e V_{T T F}^{t o t}$ is, in principle, much larger than the effect of $\left(C N L_{T T F}-E_{F}-e V_{T T F}^{t o t(0)}\right)$ on $e V_{T C N Q}^{t o t}$ (factor 0.12 vs. 0.02); this is due to the larger value of the induced density of states ( $D_{T C N Q}$ vs. $\left.D_{T T F}\right)$, see Table I and Eq. (4b). In the present case $\left(\Phi_{M}=5.3 \mathrm{eV}\right)$, however, the value of $\left(C N L_{T C N Q}-E_{F}-e V_{T C N Q}^{t o t(0)}\right)$ is small $(0.47 \mathrm{eV}$, see Fig. 2 and Table I), and its effect on $e V_{T T F}^{\text {tot }}$ is only $0.06 \mathrm{eV}$. It is remarkable that for this case, neglecting the cross terms, $0.12\left(C N L_{T C N Q}-E_{F}-e V_{T C N Q}^{\text {tot }(0)}\right)$ and $0.02\left(C N L_{T T F}-E_{F}-e V_{T T F}^{t o t(0)}\right)$, introduces an error in the potential shifts smaller than $0.10 \mathrm{eV}$. However, for the fictitious case $\Phi_{M}=1.8 \mathrm{eV}$ (see Fig. 4), that error would be as large as $-0.36 \mathrm{eV}$ for $\mathrm{eV}^{\mathrm{tot}}$ TTF .

Fig. 5 shows an energy scheme indicating how the organic energy levels are shifted with respect to the metal $\left(\Phi_{M}=5.3 \mathrm{eV}\right)$ : in a first step, we have the bare potential

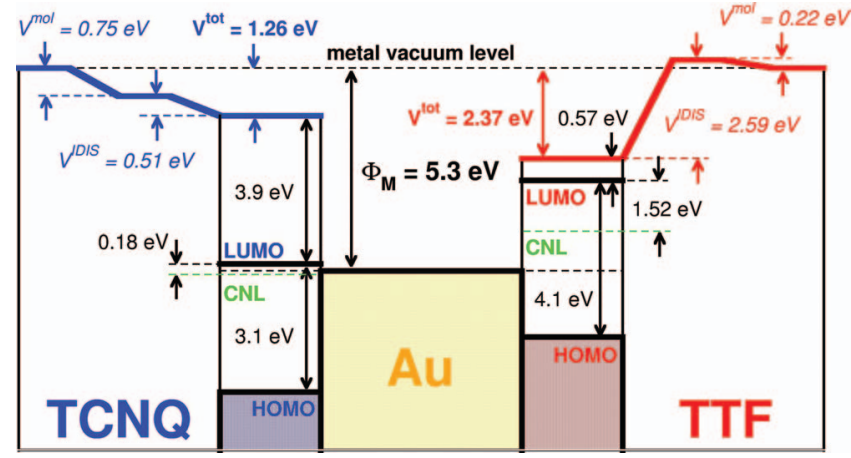

FIG. 5. Energy levels scheme indicating how the organic energy levels are shifted with respect to the metal $\left(\Phi_{\mathrm{M}}=5.3 \mathrm{eV}\right)$ after the formation of the (TTF-TCNQ)/Au(111) interface. Both molecules are adsorbed on the same $\mathrm{Au}(111)$ surface forming a mixed (TTF-TCNQ) overlayer, see Figure 1.

shifts (due to the intrinsic molecular dipoles), $e V_{T T F}^{\text {tot }(0)}$ and $e V_{T C N Q}^{\text {tot }(0)}(-0.22$ and $0.75 \mathrm{eV}$, respectively); in a second step, the charge transfer introduces new shifts, around $2.59 \mathrm{eV}$ for TTF and $0.51 \mathrm{eV}$ for TCNQ. The final shifts can be compared to the values of $e V_{T T F}^{\text {tot }}$ and $e V_{T C N O}^{\text {tot }}$ for the homogeneous TCNQ/Au(111) $)^{28}$ and TTF/Au(111) ${ }^{29}$ compact (herringbone) interfaces. In comparison with those cases, in the mixed (TTF-TCNQ)/Au(111) interface we find that $e V_{T T F}^{\text {tot }}$ and $e V_{T C N Q}^{\text {tot }}$ are reduced by, $0.32 \mathrm{eV}$ and $0.11 \mathrm{eV}$, respectively; these changes are related to an increased transfer of charge from the donor (TTF) molecules to the acceptor molecules (TCNQ) and the Au surface, as well as to changes in the potentials due to the intrinsic molecular dipoles, $e V_{T T F}^{\text {tot }(0)}$ and $e V_{T C N Q}^{t o t(0)}$. Finally, we mention that the values we obtain for the induced potentials on TTF and TCNQ, $2.37 \mathrm{eV}$ and $1.26 \mathrm{eV}$ respectively, do not support the vacuum-level pinning scenario recently suggested for organic blends on metals in the weakly interacting molecule/substrate case. ${ }^{18}$ This result allows us to discard a simplified IDIS-model in which a TTF-TCNQ pair is treated as a single molecule.

\section{The IDIS model for a general blended interface}

In Secs. III B and III C we have presented an extension of the IDIS-model to analyze the DFT results obtained for the blended TTF-TCNQ/Au(111) interface. We discuss now some general features in this approach that will be useful to analyze other blended interfaces.

We start from Eq. (4b) and calculate $\mathbf{S}$ by neglecting the product $\left(J^{e f f} D_{T C N Q}\right)\left(J^{e f f} D_{T C N Q}\right)$ as compared to $\left(1+U^{\text {eff }}\right.$ TTF $\left.D_{T T F}\right)\left(1+U^{e f f} T_{T C N Q} D_{T C N Q}\right)$ (e.g., see Table I). From now on, we replace TTF and TCNQ by the indexes 1 and 2, respectively; then, from Eq. (4b):

$$
\mathbf{S}=\left[\begin{array}{cc}
S_{1} & -\alpha_{2} S_{1}\left(1-S_{2}\right) \\
-\alpha_{1} S_{2}\left(1-S_{1}\right) & S_{2}
\end{array}\right],
$$

where $\alpha_{1}=J^{\text {eff }} / U_{1}{ }^{\text {eff }} ; \alpha_{2}=J^{\text {eff }} / U_{2}{ }^{\text {eff }} ; S_{1}=1 /\left(1+U_{1}{ }^{\text {eff }} D_{1}\right)$; and $S_{2}=1 /\left(1+U_{2}\right.$ eff $\left.D_{2}\right)$. The last two quantities, $S_{1}$ and $S_{2}$, represent the screening parameters for each independent sublayer. If we also neglect $e V_{i}^{\text {tot }(0)}$ (the potentials induced by 
the intrinsic molecular dipoles) we can write:

$$
\begin{aligned}
& e V_{1}^{t o t}=e V_{11}^{t o t}+e V_{12}^{t o t}, \\
& e V_{2}^{t o t}=e V_{21}^{t o t}+e V_{22}^{t o t},
\end{aligned}
$$

where

$$
\begin{aligned}
& e V_{11}^{\text {tot }}=\left(1-S_{1}\right)\left(C N L_{1}-E_{F}\right), \\
& e V_{12}^{\text {tot }}=a_{2} S_{1}\left(1-S_{2}\right)\left(C N L_{2}-E_{F}\right), \\
& e V_{21}^{\text {tot }}=a_{1} S_{2}\left(1-S_{1}\right)\left(C N L_{1}-E_{F}\right), \\
& e V_{22}^{\text {tot }}=\left(1-S_{2}\right)\left(C N L_{2}-E_{F}\right) .
\end{aligned}
$$

Typically, $\alpha_{1}$ and $\alpha_{2}$ are close to $1 / 2$; then, $e V_{i}^{\text {tot }}$ can be estimated using these equations that depend basically on parameters, $S_{1}, S_{2},\left(C N L_{1}-E_{F}\right)$, and $\left(C N L_{2}-E_{F}\right)$, associated with each independent component. In particular, for TTF and TCNQ, taking $S_{1}=0.22, S_{2}=0.05$, and $\alpha_{1}=\alpha_{2} \sim 1 / 2$, we practically recover Eq. (7).

Equation (9b) can be used as a fair approximation for calculating the induced potentials, $V_{i}^{\text {tot }}$, for a blended interface. It is also of interest to mention how those induced potentials appear as a function of the Fermi level position. Fig. 6(a) shows a case in which the Fermi level is below both CNLs; in this case, both $\left(C N L_{1}-E_{F}\right)$ and $\left(C N L_{2}-E_{F}\right)$ are positive, and the two components of each potential, say $e V_{11}^{\text {tot }}$ and $e V_{12}^{\text {tot }}$ (or, $e V_{21}^{\text {tot }}$ and $e V_{22}^{\text {tot }}$ ) are also positive; this is shown schematically in Figure 6(a) by indicating that the total potential, say $e V_{1}^{\text {tot }}$, is the sum of two positive contributions, $e V_{11}^{t o t}$ and $e V_{12}^{t o t}$. A second case, with $E_{F}$ in between $C N L_{1}$ and $C N L_{2}$ is also shown in Figure 6(b); in this case, because of the change in sign of $\left(C N L_{2}-E_{F}\right)$, the second component of $e V_{1}^{\text {tot }}, e V_{12}^{\text {tot }}$, changes its sign.

These two cases show that, when $E_{F}$ is below (or above) both $C N L$ s, the effect of the second component, say 2 , is to reinforce the screening effect associated with $\left(1-S_{1}\right)\left(C N L_{1}\right.$ $-E_{F}$ ). Conversely, when $E_{F}$ is in between $C N L_{1}$ and $C N L_{2}$ there appears an anti-screening effect ( $e V_{12}^{\text {tot }}$ being negative), in such a way that the second compound, say 2, tends to oppose the screening effect of $\left(1-S_{1}\right)\left(C N L_{1}-E_{F}\right)$.

These effects, depending on the position of $E_{F}$ w.r.t. $\left(C N L_{i}-E_{F}\right)$, offer a way to control the induced potentials and the interface barriers for blended organic/metal interface.

We complete this section mentioning that the effect of $e V_{i}^{\text {tot( }(0)}$ on the previous discussion is to shift the values of $\left(C N L_{i}-E_{F}\right)$ to $\left(C N L_{i}-E_{F}-e V_{i}^{t o t(0)}\right)$ as discussed above.

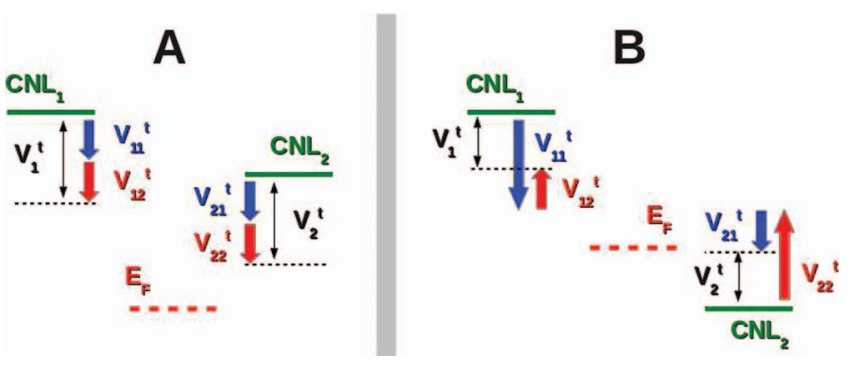

FIG. 6. Pictorial scheme showing the potentials $V_{1}^{t}$ and $V_{2}^{t}$ (including their corresponding counterparts $V_{11}^{t}, V_{12}^{t}, V_{21}^{t}$, and $V_{22}^{t}$ as described in the text) for different values of $E_{F}$ w.r.t. the charge neutrality levels $\mathrm{CNL}_{1}$ and $\mathrm{CNL}_{2}$ of the organic molecules.

\section{SUMMARY AND CONCLUSIONS}

In summary, we have analyzed the energy level alignment for the interface between a metal and an organic adlayer that consists of a blend of two different organic molecules. In particular, we have studied the (TTF-TCNQ)/Au(111) interface; this interface combines two organic molecules with strong donor (TTF) and acceptor (TCNQ) characters. The experimental evidence shows that the TTF and TCNQ molecules lie parallel to the $\mathrm{Au}(111)$ surface, forming alternating rows of TTF and TCNQ molecules. In our analysis, we start with this adlayer geometry, as suggested in Ref. 19, and relax the atomic positions by using the DFT $+\mathrm{D}$ approach. ${ }^{31,32}$

The electronic structure and energy level alignment for this interface are analyzed introducing appropriate corrections to properly describe the energy gaps for the TTF and TCNQ molecules in a local-orbital DFT calculation. These corrections are related to the charging energies $U_{\alpha}$ of the two organic molecules on the (TTF-TCNQ)/Au(111) interface; in the present calculations we have used the values for the charging energies obtained in previous calculations for the TTF/Au(111) or TCNQ/Au(111) interfaces. ${ }^{28,29}$ These calculations show that the TCNQ LUMO peak is very close to the Fermi level. Due to the interaction with the metal surface, the molecular levels are broadened, creating an important induced density of interface states in the HOMO-LUMO gap of the organic semiconductors. We find an important transfer of charge between the two organic materials, and between the organic layer and the $\mathrm{Au}(111)$ surface; in particular, we obtain $\delta n_{T C N Q}=0.19$ electrons/(TCNQ-molecule), $\delta n_{T T F}=-0.61$ electrons/(TTF-molecule) and $\delta n_{A u}=0.42$ electrons/(pair of TTF and TCNQ molecules), the important transfer of charge between the TTF and TCNQ molecules reflecting their donor and acceptor characters. The induced potentials on the TCNQ and TTF molecules are 1.26 and $2.37 \mathrm{eV}$, respectively, shifting the molecular levels downwards in energy w.r.t. the metal.

The results from the DFT calculations have been analyzed by means of an extension of the Unified-IDIS model for the case of a blended organic layer. In Sec. III this generalization is presented in detail and applied to the (TTFTCNQ)/Au(111) interface. In this case we have two different types of organic molecules in the adlayer; the basic scalar equation - Eq. (6) - relating the induced potential in the organic layer with the $C N L$ of the organic material, the screening parameter $S$ and the molecular dipole potential $e V^{\text {tot(0) }}$ is transformed into a matrix equation - Eqs. (4) and (5) - with $2 \times 2$ matrices (e.g., for $\boldsymbol{S}$ ) incorporating the effect of both organic materials. Finally, we mention that the IDIS formalism presented here can be naturally extended for more complex heterogeneous organic blend/metal interfaces involving a higher number of organic molecules.

\section{ACKNOWLEDGMENTS}

Present work was supported by Spanish MICIIN under Contract No. FIS2010-16046, the CAM under Contract No. S2009/MAT-1467, and the European Project MINOTOR (Grant No. FP7-NMP-228424). J.I.M. acknowledges funding 
from Spanish MICIIN and CSIC through Juan de la Cierva and JaeDoc Programs.

${ }^{1}$ W. R. Salanek, S. Stafstrom, and J. L. Bredas, Conjugated Polymer Surfaces and Interfaces: Electronic and Chemical Structure of Interfaces for Polymer Light Emitting Diodes (Cambridge University, Cambridge, 1996).

${ }^{2}$ N. Koch, ChemPhysChem 8, 1438 (2007).

${ }^{3}$ F. Flores, J. Ortega, and H. Vázquez, Phys. Chem. Chem. Phys. 11, 8658 (2009).

${ }^{4}$ D. Jérome and H. J. Schultz, Adv. Phys. 31, 299 (1982).

${ }^{5}$ J. R. Kirtley and J. Mannhart, Nature Mater. 7(7), 520 (2008).

${ }^{6}$ J. I. Martínez, E. Abad, C. González, F. Flores, and J. Ortega, Phys. Rev. Lett. 108, 246102 (2012).

${ }^{7}$ S. Narioka, H. Ishii, D. Yoshimura, M. Sei, Y. Ouchi, K. Seki, S. Hasegawa, T. Miyazaki, Y. Harima, and K. Yamashita, Appl. Phys. Lett. 67, 1899 (1995).

${ }^{8}$ I. G. Hill, A. Rajagopal, A. Kahn, and Y. Hu, Appl. Phys. Lett. 73, 662 (1998).

${ }^{9}$ H. Ishii, K. Sugiyama, E. Ito, and K. Seki, Adv. Mater. 11, 605 (1999).

${ }^{10}$ I. G. Hill, J. Schwartz, and A. Kahn, Org. Electron. 1, 5 (2000).

${ }^{11}$ X. Crispin, V. Geskin, A. Crispin, J. Cornil, R. Lazzaroni, W. R. Salaneck, and J. L. Brédas, J. Am. Chem. Soc. 124, 8131 (2002).

${ }^{12}$ C. Shen and A. Kahn, Org. Electron. 2, 89 (2001).

${ }^{13}$ M. Knupfer and G. Paasch, J. Vac. Sci. Technol. A 23(4), 1072 (2005).

${ }^{14}$ S. Yanagisawa and Y. Morikawa, J. Phys. Condens. Matter 21, 064247 (2009).

${ }^{15}$ P. Bagus, V. Staemmler, and C. Wöll, Phys. Rev. Lett. 89, 096104 (2002).

${ }^{16}$ G. Witte, S. Lukas, P. S. Bagus, and C. Woll, Appl. Phys. Lett. 87, 263502 (2005).

${ }^{17}$ H. Vázquez, Y. J. Dappe, J. Ortega, and F. Flores, J. Chem. Phys. 126, 144703 (2007).

${ }^{18}$ A. El-Sayed, P. Borghetti, E. Goiri, C. Rogero, L. Floreano, G. Lovat, D. J. Mowbray, J. L. Cabellos, Y. Wakayama, A. Rubio, J. E. Ortega, and D. G. de Oteyza, ACS Nano 7, 6914 (2013).

${ }^{19}$ N. González-Lakunza, I. Fernández-Torrente, K. J. Franke, N. Lorente, A. Arnau, and J. I. Pascual, Phys. Rev. Lett. 100, 156805 (2008).

${ }^{20}$ I. Fernández-Torrente, K. J. Franke, and J. I. Pascual, Phys. Rev. Lett. 101, 217203 (2008).

${ }^{21}$ HuiJuan Yan, ShanShan Li, CunJi Yan, Qing Chen, and LiJun Wan, Sci. China, Ser. B: Chem. 52(5), 559 (2009).

${ }^{22}$ H. Alves, A. S. Molinari, H. Xie, and A. F. Morpurgo, Nature Mater. 7, 574 (2008).

${ }^{23}$ J. I. Beltrán, F. Flores, J. I. Martínez, and J. Ortega, J. Phys. Chem. C 117, 3888 (2013).

${ }^{24}$ E. Abad, Y. J. Dappe, J. I. Martínez, F. Flores, and J. Ortega, J. Chem. Phys. 134, 044701 (2011).

${ }^{25}$ B. Pieczyrak, E. Abad, F. Flores, and J. Ortega, J. Chem. Phys. 135, 084702 (2011).
${ }^{26}$ E. Abad, J. Ortega, and F. Flores, Phys. Status Solidi A 209, 636 (2012).

${ }^{27}$ J. I. Martínez, E. Abad, F. Flores, J. Ortega, and G. Brocks, Chem. Phys. 390, 14 (2011)

${ }^{28}$ J. I. Martínez, E. Abad, F. Flores, and J. Ortega, Phys. Status Solidi B 248, 2044 (2011).

${ }^{29}$ J. I. Martínez, E. Abad, C. González, J. Ortega, and F. Flores, Org. Electron. 13, 399 (2012).

${ }^{30}$ V. G. Ruiz, W. Liu, E. Zojer, M. Scheffler, and A. Tkatchenko, Phys. Rev. Lett. 108, 146103 (2012).

${ }^{31}$ S. Grimme, J. Comput. Chem. 27, 1787 (2006).

${ }^{32}$ P. Giannozzi, S. Baroni, N. Bonini, M. Calandra, R. Car, C. Cavazzoni, D. Ceresoli, G. L. Chiarotti, M. Cococcioni, I. Dabo, A. Dal Corso, S. de Gironcoli, S. Fabris, G. Fratesi, R. Gebauer, U. Gerstmann, C. Gougoussis, A. Kokalj, M. Lazzeri, L. Martin-Samos, N. Marzari, F. Mauri, R. Mazzarello, S. Paolini, A. Pasquarello, L. Paulatto, C. Sbraccia, S. Scandolo, G. Sclauzero, A. P. Seitsonen, A. Smogunov, P. Umari, and R. M. Wentzcovitch, J. Phys.: Condens. Matter 21, 395502 (2009).

${ }^{33}$ Y. Zhang and W. Yang, Phys. Rev. Lett. 80, 890 (1998).

${ }^{34}$ V. Barone, M. Casarin, D. Forrer, M. Pavone, M. Sambi, and A. Vittadini, J. Comput. Chem. 30, 934 (2009).

${ }^{35}$ M. Elstner, P. Hobza, T. Frauenheim, S. Suhai, and E. Kaxiras, J. Chem. Phys. 114, 5149 (2001).

${ }^{36}$ A. Gavezzotti, J. Am. Chem. Soc. 105, 5220 (1983); J. D. Dunitz and A. Gavezzotti, Acc. Chem. Res. 32, 677 (1999); G. Filippini and A. Gavezzotti, Acta Crystallogr., Sect. B: Struct. Sci. 49, 868 (1993).

${ }^{37}$ D. J. Chadi and M. L. Cohen, Phys. Rev. B 8, 5747 (1973).

${ }^{38}$ J. P. Lewis, P. Jelínek, J. Ortega, A. A. Demkov, D. G. Trabada, B. Haycock, H. Wang, G. Adams, J. K. Tomfohr, E. Abad, H. Wang, and D. A. Drabold, Phys. Status Solidi B 248, 1989 (2011).

${ }^{39}$ J. P. Lewis, K. R. Glaesemann, G. A. Both, J. Fritsch, A. A. Demkov, J. Ortega, and O. F. Sankey, Phys. Rev. B 64, 195103 (2001).

${ }^{40}$ P. Jelínek, H. Wang, J. P. Lewis, O. F. Sankey, and J. Ortega, Phys. Rev. B 71, 235101 (2005).

${ }^{41}$ A. A. Demkov, J. Ortega, O. F. Sankey, and M. P. Grumbach, Phys. Rev. B 52, 1618 (1995).

${ }^{42}$ F. J. García-Vidal, J. Merino, R. Pérez, R. Rincón, J. Ortega, and F. Flores, Phys. Rev. B 50, 10537 (1994).

${ }^{43}$ M. A. Basanta, Y. J. Dappe, P. Jelínek, and J. Ortega, Comput. Mater. Sci. 39, 759 (2007).

${ }^{44}$ M. Fuchs and M. Scheffler, Comput. Phys. Commun. 119, 67 (1999).

${ }^{45}$ For $\mathrm{C}, \mathrm{N}, \mathrm{S}$, and Au the basis set consists of 9 numerical atomic-like orbitals per atom: $1 \mathrm{~s}$ orbital, $3 \mathrm{p}$ orbitals $\left(\mathrm{p}_{\mathrm{x}}, \mathrm{p}_{\mathrm{y}}\right.$, and $\left.\mathrm{p}_{\mathrm{z}}\right)$ and $5 \mathrm{~d}$ orbitals, see Ref. 43.

${ }^{46} \mathrm{~F}$. Flores and J. Ortega, The Molecule-Metal Interface, edited by N. Koch, N. Ueno, and A. T. S. Wee (Wiley-VCH, 2013), Chap. 2.

${ }^{47}$ F. Flores, E. Abad, J. I. Martínez, B. Pieczyrak, and J. Ortega, J. Phys.: Condens. Matter 25, 094007 (2013).

${ }^{48}$ E. Abad, C. González, J. Ortega, and F. Flores, Org. Electron. 11, 332 (2010) 